\title{
Desempenho do algoritmo SEBAL para estimativa de vazão em bacias hidrográficas do Cerrado Brasileiro
}

\author{
Performance of the SEBAL algorithm for flow estimation in watersheds of \\ Brazilian Savannah
}

\author{
Alécio Perini Martins 1 \\ Emerson Galvani ${ }^{2}$
}

\begin{abstract}
Palavras-chave
Modelagem ambiental

Geotecnologias

Balanço de Radiação

Evapotranspiração
\end{abstract}

\begin{abstract}
Resumo
O estudo das variáveis componentes do Balanço de Radiação, bem como valores derivados de fluxo de calor e evapotranspiração são fundamentais em climatologia, principalmente para compreender a dinâmica do ciclo hidrológico. A diminuição das chuvas pode estar relacionada à mudança nos valores de evapotranspiração real em superfície, visto que mudanças no uso da terra como a conversão de florestas em pastagens alteram o albedo, o índice de vegetação e a temperatura de superfície. Neste sentido, estudos com foco nos efeitos das mudanças ambientais sobre os fluxos de energia na superfície são fundamentais para a conservação dos recursos hídricos e, consequentemente, da vida na Terra. A pesquisa tem como objetivo avaliar o desempenho do algoritmo SEBAL (Surface Energy Balance Algorithms for Land) para a estimativa de vazão anual em bacias hidrográficas situadas no Cerrado Brasileiro considerando esta ser o resultado simplificado da subtração entre os valores de precipitação e evapotranspiração real. Permite, deste modo, a estimativa de vazão para bacias hidrográficas que não dispõem de pontos de monitoramento, contribuindo com estudos sobre disponibilidade hídrica em regiões extensas e de difícil acesso. Os resultados demonstraram correlação de 0,99 e coeficiente de determinação de 0,99 entre vazões anuais estimadas de acordo com as categorias de uso e cobertura da terra e vazões observadas em estações fluviométricas, com desempenho de 99\%, constatando a aplicabilidade do modelo para estimativa de vazões em áreas sem pontos de monitoramento. Para as vazões mensais estimadas a partir de informações registradas por estações automáticas do INMET, nota-se considerável efeito da sazonalidade, com registro de valores negativos no período seco. Apesar disso, as vazões estimadas apresentaram correlação de 0,80 com os valores observados, com desempenho de $89 \%$. Em média, as vazões nas bacias estudadas corresponde a $20 \%$ do total do volume precipitado apresentando subestimativa em relação aos valores aferidos em campo, com erro médio de $-0,5898 \mathrm{~m}^{3} / \mathrm{s} / \mathrm{m}^{2}$.
\end{abstract}

\footnotetext{
1 Unidade Acadêmica Especial de Estudos Geográficos - Universidade Federal de Jataí. alecioperini@ufg.br ${ }^{2}$ Departamento de Geografia - Faculdade de Filosofia, Letras e Ciências Sociais - Universidade de São Paulo. egalvani@usp.br
} 


\section{Keywords:}

Environmental modeling

Geotechnologies

Radiation Balance

Evapotranspiration

\section{Abstract}

Studies of the variables making up the radiation balanceare essential in climatology, mainly to understand the dynamics of the hydrologic cycle. Decreases in rainfall can be linked to changes in actual evapotranspiration values on the surface, since changes in land use modify the albedo, the vegetation index, and the surface temperature. The objective of the present study was to evaluate the performance of the Surface Energy Balance Algorithm for Land algorithm in estimating the annual flow in watersheds located in the Brazilian savanna, considered as the simplified result of subtracting the values of rainfall from actual evapotranspiration. The algorithm allows estimation of flows for watersheds that do not have monitoring points, contributing to studies of water availability in large regions that are difficult to access. The results showed a correlation coefficient of 0.99 and a coefficient of determination of 0.99 between annual flows estimated according to categories of use and coverage of land and flows observed at fluviometric stations, with a performance of $99 \%$, indicating the applicability of the model to estimate flows in areas without monitoring points. For the monthly flows estimated from information recorded by INMET automatic stations, there is a considerable effect of seasonality, with a record of negative values in the dry period. Despite this, the estimated flows showed a correlation of 0.80 with the observed values, with a performance of $89 \%$. On average, flows in the studied watersheds account for $20 \%$ of total rainfall volume, which is an underestimate in comparison with values measured in the field, with an average error of $0.5898 \mathrm{~m}^{3} / \mathrm{s} / \mathrm{m}^{2}$.

\section{INTRODUÇÃO}

O estudo das variáveis componentes do Balanço de Energia, bem como valores derivados de fluxo de calor e evapotranspiração são fundamentais para compreender a dinâmica do ciclo hidrológico. Essas estimativas tornam possível, por exemplo, planejar atividades de irrigação, estimar necessidade de reposição de água no solo e desenvolver estudos de recarga de água em subsuperfície, além de permitir a compreensão de mudanças climáticas e ambientais.

O crescimento desordenado dos centros urbanos e a intensa atividade industrial contribuem de forma direta com o agravamento da crise hídrica, porém, a maior pressão sobre os recursos hídricos, que interfere diretamente na produção e oferta de água, é exercida pelas práticas agropecuárias. Mudanças bruscas nas relações de uso e cobertura das terras, em especial pela supressão de florestas, e a ocupação desordenada de fundos de vale e áreas de recarga de aquíferos, entre outros, interferem diretamente no ciclo hidrológico, reduzindo a capacidade do sistema de "produzir" água e, consequentemente, a oferta deste recurso (REBOUÇAS, 1997; MARTINS; GOMES FILHO, 2013; CUNHA; ALVALÁ; OLIVEIRA, 2013).

A diminuição das chuvas pode ser relacionada diretamente à mudança nos valores de evapotranspiração real em superfície, visto que mudanças no uso da terra, como a conversão de florestas em pastagens, alteram o albedo, o índice de vegetação e a temperatura de superfície, parâmetros biofísicos essenciais ao balanço de radiação e, consequentemente, na definição de fluxos de calor e evapotranspiração (CUNHA; ALVALÁ; OLIVEIRA, 2013). Neste sentido, estudos com foco nos efeitos das mudanças ambientais sobre os fluxos de energia na superfície são fundamentais para a conservação dos recursos hídricos e, consequentemente, da vida na Terra.

Popularmente conhecido como "o pai das águas”, é no Cerrado que se localizam grande parte das nascentes que originam as principais bacias hidrográficas do país (Rios Paraná, São Francisco, Araguaia, Tocantins, entre outros). Segundo maior domínio morfoclimático do Brasil, o Cerrado estende-se por mais de 2 milhões de $\mathrm{km}^{2}$ (aproximadamente 24\%) do território nacional e encontra-se em acelerado processo de degradação desde meados do século XX. Klink e Machado (2005) destacaram que, apesar da grande biodiversidade, apenas $2,2 \%$ do Cerrado encontrava-se protegido em unidades de proteção integral e 1,9\% em unidades de uso sustentável, enquanto que terras indígenas correspondiam a 4,1\% da área. 
Machado et. al. (2004, p.6-7) destacam que entre 1985 e 1993 a perda média anual de áreas de Cerrado foi de 1,5\%, caindo para $0,67 \%$ entre 1993 e 2002, estimando-se uma taxa anual de desmatamento de aproximadamente $1,1 \%$ a ano. Os autores estimaram, ainda, que neste ritmo seria de se esperar que o Cerrado desaparecesse até o ano de 2030. Estudos posteriores como os de Rocha et. al. (2011), apontam um desmatamento de 366.100 ha entre os anos de 2002 e 2009 , com $47 \%$ dos alertas concentrados nos estados de Mato Grosso e Bahia, demonstrando que a expansão da fronteira agrícola no Cerrado continua em plena atividade, sobretudo na divisa entre os estados de Goiás e Mato Grosso, na linha de contato entre Cerrado e Amazônia e na região conhecida como MATOPIBA, nas fronteiras dos estados do Maranhão, Tocantins, Piauí e Bahia. De acordo com dados do PRODES/INPE (2019), entre os anos de 2008 e 2019, o Cerrado apresentou desmatamento 35\% superior ao da Amazônia. Todos os estados apresentaram reduções progressivas das áreas de Cerrado, com destaque para Mato Grosso, Goiás e Minas Gerais.

Assim, é fundamental o desenvolvimento de pesquisas que subsidiem políticas públicas e planos de ação que visem a recuperação de áreas de Cerrado e a "produção de água" no âmbito de bacias hidrográficas. Entre estas ações está a criação de unidades de conservação, restauração da vegetação de baixo albedo, aumento da rugosidade da superfície favorecendo a retenção e infiltração da água das chuvas, diminuição do escoamento superficial, entre outros. Lima (2010) destaca que a "produção de água" refere-se à descarga total da bacia em determinado período de tempo (Q), sendo, de forma simplificada, a fração da precipitação que não retornou ao ciclo em forma de evapotranspiração $(\mathrm{Q}=\mathrm{P}-\mathrm{ET})$.

Este conjunto de ações ajudam no "controle" da evapotranspiração ao mesmo tempo em que garantem a regularização das vazões, principalmente em áreas com sazonalidade tão marcante e longos períodos de estiagem como no Cerrado. Atualmente, uma infinidade de técnicas encontra-se a disposição para mensurar estas relações de "produção e perda" de água no ambiente. Em superfície, a Agência Nacional de Águas (ANA) dispõe de uma rede de observação de chuvas e vazões que recobre razoavelmente o território nacional, além da existência de postos de monitoramento de agências estaduais, ONG's e empresas de produção de energia. O Instituto Nacional de Meteorologia dispõe de uma rede de estações que fornecem não apenas dados pluviométricos, mas informações de temperatura, umidade, ventos e pressão que permitem calcular a evapotranspiração por diferentes métodos e modelos.

Entre os diversos modelos climatohidrológicos utilizados para a estimativa da evapotranspiração, como por exemplo, o SVAT (Soil-Vegetation-Atmosphere Transfer Model) e o METRIC (Mapping evapotranspiration at hight resolution and with internalized calibration), optou-se pelo uso do SEBAL (Surface Energy Balance Algorithms for Land), principalmente, em decorrência da extensão da área de estudo, da escassez de dados climatológicos e da facilidade de operação do modelo, que necessita de poucas informações de campo para estimar a evapotranspiração (PEREIRA; SEDIYAMA; VILLA NOVA, 2013; MARTINS, 2015; ALVES, 2019).

O SEBAL, desenvolvido por Bastiaanssen (1995), é um dos mais completos modelos para estudos de fluxo de calor na superfície com o uso de imagens de satélite e algumas informações climatológicas, como temperatura do ar e velocidade dos ventos. Essencialmente matemático, o modelo evita o emprego de suposições, diminuindo a margem de erro em sua validação. Liou e Kar (2014, p. 2831) destacam que o SEBAL foi projetado para calcular os componentes do balanço de energia em escalas local e regional, com poucos dados de superfície, usando relações empíricas e parametrização física. Sob várias condições climáticas, foi verificado nas escalas de campo precisões de $85 \%$ e $95 \%$ nas escalas diária e sazonal, respectivamente, em mais de 30 países.

De acordo com Bastiaanssen (1995), o desenvolvimento do SEBAL teve como principal estímulo superar problemas apresentados por alguns algoritmos na estimativa de fluxos de superfície a partir de imagens de sensoriamento remoto. Bastiaanssen et al. (1998), destacam que os principais problemas apresentados pelo SEBAL relacionam-se à questão de os dados serem instantâneos, o que tornam as estimativas diárias e sazonais relativamente deficientes para escalas mais detalhadas.

O SEBAL vem sendo utilizado por vários pesquisadores no mundo todo para diversos agrossistemas, destacando além dos estudos de Bastiaanssen (1995; 2000) e Bastiaanssen et al. (1998), as publicações de Allen et. al. (2002), Ahmad e Bastiaanssen (2003), Ayenew (2003), Hemakumara et al (2003), Tasumi et al (2005), Giacomoni (2008), Bezerra (2008) Kongo e Jewitt (2006), Kimura et al (2007), Mendonça (2007), Nicácio (2008), Gomes (2009), Li e Whenzi (2010), Sun et. al. (2011), Ruhoff (2011), Martins 


\section{(2015) e Freitas (2018).}

Assim, a pesquisa tem como objetivo avaliar o desempenho do algoritmo SEBAL no cálculo da evapotranspiração real e, por meio do balanço hídrico simplificado, para estimativa de vazão anual em bacias hidrográficas situadas no Cerrado Brasileiro. Permite, deste modo, a estimativa de vazão para bacias hidrográficas que não dispõem de pontos de monitoramento, contribuindo com estudos sobre disponibilidade hídrica em regiões extensas e de difícil acesso.

\section{ÁREA DE ESTUDO}

Considerando a diversidade de paisagens da região, foram selecionadas sete bacias hidrográficas ou parte de bacias em região com características climáticas similares, sendo elas
(Figura 1): a) Bacia do Rio Formoso, com $1.532 \mathrm{~km}^{2}$ de área, localizada no extremo sudoeste do estado de Goiás e com mais de $40 \%$ do território representado pelo Parque Nacional das Emas; b) Alto curso da Bacia do Rio Sucuriú $\left(3.775 \mathrm{~km}^{2}\right)$, na região nordeste de Mato Grosso do Sul, com grande diversidade de usos agropecuários; c) Alto e médio cursos das bacias dos rios Doce $\left(2.015 \mathrm{~km}^{2}\right)$, Monte Alegre (809 $\mathrm{km}^{2}$ ), Verdão $\left(1.015 \mathrm{~km}^{2}\right)$ e Verdinho $\left(1.668 \mathrm{~km}^{2}\right)$, entre os municípios de Jataí, Rio Verde e Montividiu no Sudoeste de Goiás, com grande diversidade de áreas agropecuárias, cultivo de cana-de-açúcar e agricultura irrigada por pivô central e reduzidas áreas de vegetação remanescente; d) Bacia do Rio Bonito (2.015 $\mathrm{km}^{2}$ ), nos municípios de Caiapônia, Palestina de Goiás e Arenópolis, em região de expansão da fronteira agrícola goiana.

Figura 1 - Localização da área de estudo.

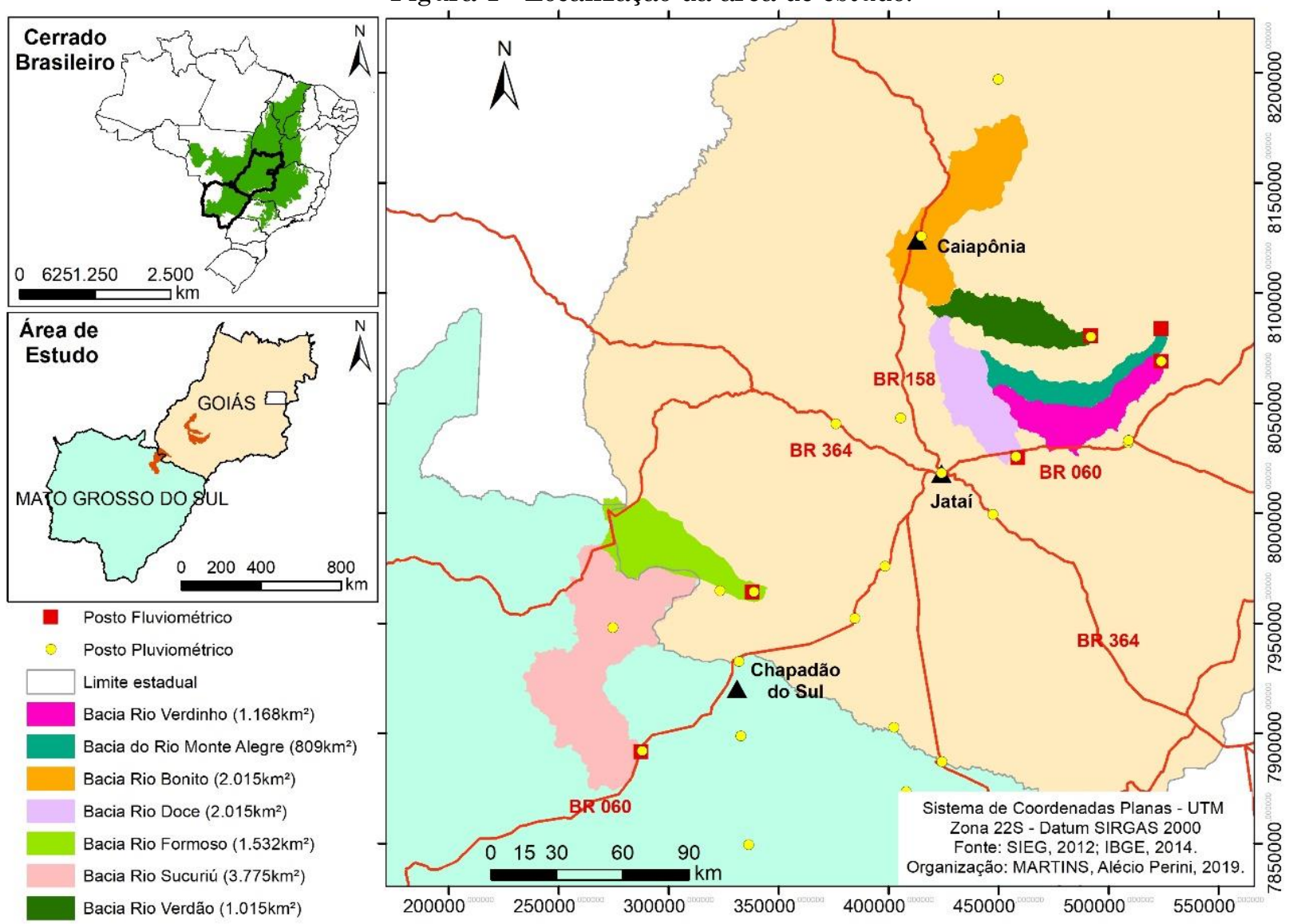

Fonte: SIEG, 2012; IBGE, 2014. Org.: MARTINS, 2019.

Uma das principais características da área de estudo é a topografia plana e suavemente ondulada, fundamental para que o Sudoeste de Goiás se tornasse foco dos programas de desenvolvimento agrícola federais nas décadas de 1970 e 1980. Martins et. al. (2016) sintetizam a paisagem da região em duas unidades bastante distintas: as chapadas em relevo plano esculpido em basalto e recoberto por arenitos e sedimentos terciários que dão origem a latossolos; e as depressões e vales, com relevo suave-ondulado a ondulado, com presença de arenitos mais antigos 
e solos de textura arenosa, originalmente recobertos por diferentes feições de cerrados.

O clima é do tipo Aw, segundo a classificação de Köppen, com mais de $85 \%$ das chuvas concentradas entre os meses de outubro e abril e estação seca pronunciada de maio a setembro, destacando a forte sazonalidade (MARIANO, 2005).

\section{PROCEDIMENTOS}

Bases de dados e implementação do modelo SEBAL para estimativa de evapotranspiração

Inicialmente, foram identificadas três estações meteorológicas do INMET na região para calibração e validação do modelo: Jataí (GO), Caiapônia (GO) e Chapadão do Sul (MS). As informações necessárias para calibração do modelo são: temperatura do ar média, máxima e mínima $\left({ }^{\circ} \mathrm{C}\right)$; umidade relativa do ar máxima e mínima (\%); pressão atmosférica (hPa); velocidade dos ventos $(\mathrm{m} / \mathrm{s})$; precipitação $(\mathrm{mm})$ e radiação $\left(\mathrm{W} / \mathrm{m}^{2}\right)$.

$\mathrm{O}$ estudo foi desenvolvido para o ano hidrológico de 01/10/2018 a 30/09/2019 a partir de dados de precipitação e vazão obtidos junto aos postos pluviométricos e fluviométricos da ANA localizados nos municípios de Jataí, Rio Verde, Montividiu, Caiapônia, Arenópolis, Serranópolis, Aporé e Chapadão do Céu (Goiás), e Chapadão do Sul, Cassilândia e Costa Rica (Mato Grosso do Sul). O banco de imagens foi composto por imagens de satélite (Landsat 8, sensores OLI e TIRS) e de radar (SRTM), todas com resolução espacial de $30 \mathrm{~m}$. Entre as imagens de satélite, foram utilizados os sensores OLI (bandas 2, 3, 4, 5, 6, 7 e 8) e TIRS (banda 10) do Landsat 8 obtidas gratuitamente mediante cadastro na página do Serviço Geológico dos Estados Unidos (USGS).

As etapas da pesquisa foram desenvolvidas no software ArcGIS 10.6.1®, licenciado para o Laboratório de Geoinformação da UFG/Regional Jataí, utilizando principalmente a Calculadora Raster. Inicialmente, procedeu-se com a correção radiométrica de cada banda a partir dos cálculos de Radiância e Reflectância conforme descrito por Ariza (2013). Os cálculos relativos ao ângulo de incidência da radiação, distância terra-sol, radiação solar exoatmosférica, reflectância corrigida e transmissividade atmosférica seguiram os parâmetros do modelo METRIC descritos por Allen et. al. (2007).

Identificou-se a correlação existente entre vazões estimadas e vazões observadas, sendo que para aquelas que apresentaram valores superiores a 0,7 (correlação forte), foram calculadas as equações de regressão, o erro médio, o erro quadrático, além da realização de testes de desempenho pelos coeficientes D e C. Ambos apresentam uma escala de desempenho que varia de péssimo a ótimo, conforme Oliveira (2016): Péssimo < 0,40; Mau 0,40 a 0,50; Sofrível 0,50 a 0,$60 ;$ Mediano 0,60 a 0,65; Bom 0,65 a 0,70 ; Muito bom 0,75 a 0,85; e Ótimo > 0,85. O desempenho de acordo com o Índice $\mathrm{D}$ relacionase ao afastamento dos valores preditos com relação aos observados (WILLMOTT et. al, 1985), enquanto que o Índice $\mathrm{C}$ reflete a confiança e/ou desempenho do método, considerando que este é obtido pela multiplicação do valor de correlação de Pearson pelo Índice D (CAMARGO; SENTELHAS, 1997).

A implementação do modelo SEBAL seguiu os procedimentos descritos por Allen et. al. (2002), atualizados por diversas pesquisas desenvolvidas por Ahmad e Bastiaanssen (2003), Ayenew (2003), Tasumi et al (2005), Bezerra (2006) Kongo e Jewitt (2006), Kimura et al (2007), Mendonça (2007), Nicácio (2008) e Martins (2016).

Para o cálculo da Evapotranspiração de Referência, utilizou-se a equação de Penman Monteith descrita por Allen et. al. (1998) no FAO Irrigation and drainage paper 56. A evapotranspiração real instantânea (ETh) foi calculada pixel a pixel, considerando tanto condições ideais, quanto de saturação ou restrição hídrica, a partir do saldo de calor latente $(\lambda \mathrm{ET})$ e o calor latente de evaporação $(\lambda)$, conforme equações 1 e 2, descritas por Allen et. al. (2007).

$E T h=3600 * \lambda \mathrm{ET} / \lambda$

Onde $\lambda$ corresponde ao calor latente de evaporação (equação 2, dada em $\mathrm{J} / \mathrm{Kg}$ ), calculado com base na Temperatur de Superfície (Ts) e 3600 é o valor de conversão de segundos para horas (BASTIAANSSEN et al, 1998; NICÁCIO, 2008).

$$
\lambda=[2,501-0,00236 *(\mathrm{Ts}-273,16)] * 10^{6}
$$

Para estimar a evapotranspiração diária (equação 3) e mensal/sazonal (equação 4) é necessário calcular os valores de referência (ETo) para 24 horas e para o período, além de um componente conhecido como Fração Evaporativa, que é definida como a razão entre a evapotranspiração instantânea (ETh) e a 
evapotranspiração de referência (ETo). De acordo com Allen et. al. (2002), os valores da fração evaporativa (ETrF) são semelhantes ao coeficiente da cultura (Kc), variando de 0 a 1 e, ocasionalmente, atingindo valores superiores a 1 quando ETh for significativamente superior à ETo, como em corpos hídricos e áreas com vegetação arbórea muito densa.

$$
E T 24=E T r F * E T o 24
$$

Onde ETo24 (mm/dia) é o acumulado de ETo em 24 horas para o dia de obtenção da imagem, calculado a partir da soma dos valores de ETo horários ao longo do dia.

$$
\text { ETperiodo }=\text { ETrFperiod } * \sum_{1}^{n} \text { ETo } 24
$$

De acordo com Allen et al (2002), o SEBAL calcula a evapotranspiração diária assumindo que a $\mathrm{ETrF}$ instantânea é a mesma que a média de 24 horas.

\section{Mapeamento de uso e cobertura da terra e estimativa de vazão anual}

Para o mapeamento das categorias de uso e cobertura da terra selecionou-se duas imagens OLI/Landsat 8 sem cobertura de nuvens para cada uma das órbita/ponto utilizadas no estudo: a) Para a órbita/ponto 223/72 imagens dos dias 02/02/2019 (verão) e 26/06/2019 (inverno); b) Para a órbita/ponto 224/73 imagens do dia 08/01/2019 (verão) e 19/07/2019 (inverno). São necessárias imagens de períodos diferentes considerando a sazonalidade da região, sendo necessário identificar os diferentes tipos de cultivo (grãos, cana-de-açúcar e áreas irrigadas).

Inicialmente, gerou-se a composição colorida considerando as bandas $2,3,4,5,6$ e 7 do OLI/Landsat 8 e posterior fusão com a banda 8 (pancromática com 15 metros de resolução espacial) para geração de imagem colorida com resolução reamostrada para 15 metros. Embora já apresentem georreferenciamento, este foi conferido e corrigido a partir da malha viária e das redes de drenagem em escala de 1:100.000 disponibilizadas pelo IBGE. As imagens de satélite foram obtidas gratuitamente na página do Serviço Geológico dos Estados Unidos (USGS/Earth Explorer) e processadas no software ArcGIS 10.6.1 ® licenciado para o Laboratório de Geoinformação da UFG/Regional Jataí.

A classificação das cenas foi realizada de forma supervisionada, com a criação de 50 amostras de cada uma das classes: a) Agricultura (cana); b) Agricultura (grãos); c) Agricultura irrigada; d) Água; e) Área úmida; f) Cerrado; g) Cerradão/Formações florestais; h) Pastagem; i) Pastagem degradada; j) Silvicultura; k) Solo descoberto/ área urbana/ estradas pavimentadas. Foi aplicado o classificador Interactive Supervised Classification, disponível na barra de ferramentas Image Classification. Por fim, as imagens temáticas foram convertidas para formato de polígono, passaram por edição manual de erros e validação kappa a partir de pontos selecionados no aplicativo Google Earth PRO, com nível de acerto de $90 \%$.

O mapa de uso e cobertura foi convertido novamente em formato raster, recortado para as áreas das sete bacias hidrográficas e os pontos usados para validação kappa foram cruzados com as variáveis obtidas nas diversas etapas de implementação do SEBAL por meio da ferramenta Zonal Statistics as a Table, onde foram calculados os valores médios, máximos, mínimos e desvios padrão de cada grupo de amostras.

Para a estimativa de vazão (Q), considerouse a relação: $\mathrm{Q}=\mathrm{P}-\mathrm{ET}$, onde $\mathrm{P}$ corresponde à precipitação média na bacia (mensal e anual) obtidas pelas estações pluviométricas da ANA, e ET corresponde à evapotranspiração mensal estimada com o uso do SEBAL a partir de produtos de sensoriamento remoto. Para a evapotranspiração considerou-se o valor mensal estimado para o pixel da estação meteorológica automática do INMET, usada para cálculo da Evapotranspiração de Referência (ETo) e o valor mensal a partir da evapotranspiração de cada categoria de uso e cobertura da terra.

No cálculo da vazão anual considerando as categorias de uso e cobertura da terra, seguiu-se as seguintes etapas: a) Cálculo da área de cada categoria de uso e cobertura da terra em $\mathrm{m}^{2}$; b) Estimativa de evapotranspiração anual de cada categoria de uso, em mm/ano; c) Cálculo da média pluviométrica anual da bacia, em mm/ano; d) Subtração dos valores de evapotranspiração do total pluviométrico, sendo o resultado considerado como a vazão (em mm/ano) de cada categoria de uso e cobertura da terra; e) Multiplicação dos valores de vazão pela área de cada categoria de uso, com resultado em $\mathrm{mm} / \mathrm{ano}^{*} \mathrm{~m}^{2}$; f) Conversão dos valores obtidos na etapa anterior para $\mathrm{m}^{3} / \mathrm{ano} / \mathrm{m}^{2} ; \mathrm{g}$ ) Conversão dos valores da etapa anterior para dados diários, horários e, por fim, em $\mathrm{m}^{3} / \mathrm{s} / \mathrm{m}^{2}$.

Somados, os valores de vazão por segundo por área de cada categoria de uso e cobertura da terra correspondem à vazão da bacia em um ano hidrológico que, nesta pesquisa foi de 01/10/2018 
a 30/09/2019. Os resultados foram conferidos com os dados observados nas estações fluviométricas da ANA e passaram por análise estatística.

\section{RESULTADOS E DISCUSSÃO}

Conforme observado na Tabela 1 , as vazões estimadas pelo balanço hídrico simplificado (P-
ETr) correspondem a aproximadamente 20\% do volume precipitado no decorrer do ano hidrológico 2018/2019, incluído como ano padrão por apresentar valores próximos às médias pluviométricas registradas na região. Nas bacias dos Rios Formoso e Sucuriú as precipitações apresentaram-se pouco acima da média para a região devido a valores elevados registrados no mês de novembro de 2018 .

Tabela 1. Variáveis aferidas nas estações meteorológicas do INMET, estações fluviométricas e pluviométricas da ANA e a partir das categorias de uso e cobertura da terra - 2019*.

\begin{tabular}{|c|c|c|c|c|c|c|}
\hline $\begin{array}{c}\text { Variáveis/ Bacias } \\
\text { hidrográficas }\end{array}$ & Formoso & Doce & Sucuriú & Verdão & $\begin{array}{l}\text { Monte } \\
\text { Alegre }\end{array}$ & Verdinho \\
\hline Temp. média $\left({ }^{\circ} \mathrm{C}\right)$ & 23,2 & 23,0 & 23,2 & 23,0 & 23,0 & 23,0 \\
\hline Precipitação (mm) & 1897,5 & 1641,2 & 1897,5 & 1620,3 & 1497,2 & 1541,0 \\
\hline Eto $(\mathbf{m m})$ & 2096,9 & 2000,9 & 2096,9 & 2049,5 & 2049,5 & 2049,0 \\
\hline $\operatorname{Etr}(\mathbf{m m})$ & 1480,3 & 1316,4 & 1361,6 & 1334,1 & 1230,2 & 1223,0 \\
\hline P-Etr (mm) & 402,8 & 324,8 & 441,0 & 286,2 & 267,0 & 318,2 \\
\hline Percentual P-ETR/P (\%) & 21,2 & 19,8 & 23,2 & 17,7 & 17,8 & 20,6 \\
\hline Área $\left(\mathbf{k m}^{2}\right)$ & 1532,0 & 2015,0 & 3775,0 & 1015,0 & 809,0 & 1168,0 \\
\hline $\begin{array}{c}Q \text { estimada - estação } \\
\left(\mathrm{m}^{3} / \mathrm{s} / \mathrm{m}^{2}\right)\end{array}$ & 21,4 & 21,3 & 27,2 & 9,2 & 7,2 & 11,6 \\
\hline Q estimada - uso $\left(\mathrm{m}^{3} / \mathrm{s} / \mathrm{m}^{2}\right)$ & 23,6 & 21,1 & 78,0 & 12,7 & 10,9 & 15,9 \\
\hline $\begin{array}{c}\text { Q observada - ANA } \\
\left(\mathrm{m}^{3} / \mathrm{s} / \mathrm{m}^{2}\right)\end{array}$ & 24,9 & 23,8 & 74,7 & 14,2 & 11,5 & 16,6 \\
\hline
\end{tabular}

Nesta tabela não são apresentadas informações sobre o Rio Bonito, que não apresenta posto de monitoramento fluviométrico. Org.: Dos autores, 2019.

Nota-se que os valores brutos de vazão anual estimados considerando as categorias de uso e cobertura da terra são semelhantes aos valores observados nos postos pluviométricos da ANA, mas quando se observam os valores estimados para a estação automática do INMET as variações são significativas. Isso acontece em função da marcante sazonalidade do Cerrado, que apresenta estação seca entre os meses de maio e setembro. Para estes períodos, os valores estimados de vazão foram negativos (Figura 2) para a estação do INMET, indicando déficit hídrico.
As vazões estimadas a partir das categorias de uso e cobertura da terra apresentaram correlação muito forte com as mensuradas nas estações fluviométricas da ANA, com valores de 0,9991 e coeficiente de determinação de 0,9969 que corresponde a uma eficiência de quase 100\% do modelo (Tabela 2 e Figura 3). Quando comparados os dados observados à vazão estimada considerando as estações automáticas do INMET a correlação foi de 0,8099 e o coeficiente de determinação de 0,6556 demonstrando, assim como os gráficos da figura 2, o efeito da sazonalidade sobre a eficiência da estimativa. 
Figura 2 - Diagramas de dispersão demonstrando a relação entre vazão observada e vazão estimada considerando as estações automáticas do INMET ano hidrológico 2018/2019. Na sequência, apresentamse os dados das bacias hidrográficas dos rios Formoso (A), Monte Alegre (B), Doce (C), Sucuriú (D), , Verdão (E) e Verdinho (F).
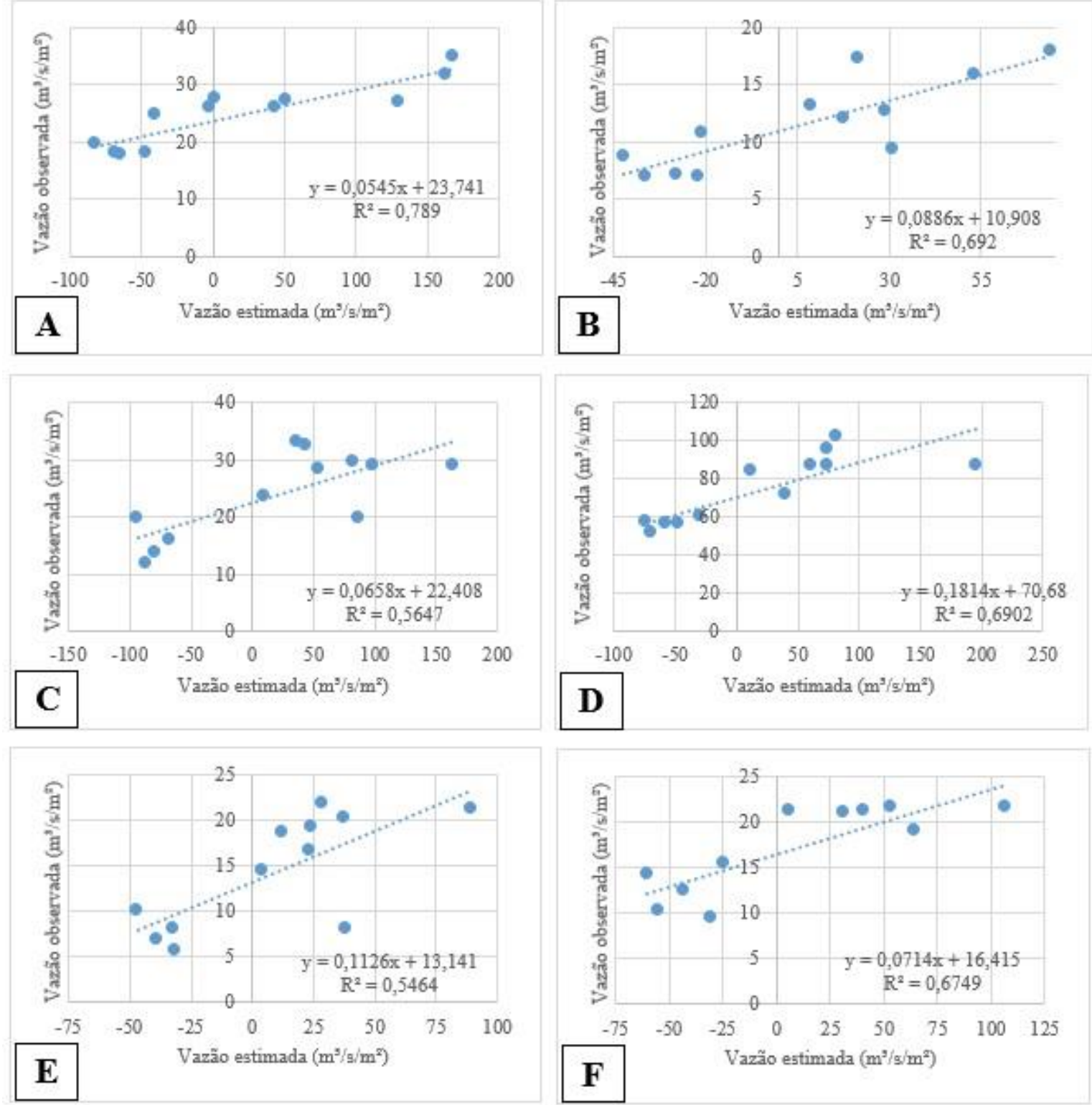

Fonte: INMET, 2019; ANA, 2019. Org.: dos autores, 2019.

Tabela 2. Estatística de validação de parâmetros estimados e de referência no ano hidrológico $2018 / 2019$.

\begin{tabular}{lcccccc}
\hline Variáveis & $\mathbf{R}$ & $\mathbf{R}^{\mathbf{2}}$ & $\mathbf{E Q M}$ & $\mathbf{R E Q M}$ & $\begin{array}{c}\text { Índice } \\
\mathbf{D}\end{array}$ & $\begin{array}{c}\text { Índice } \\
\mathbf{C}\end{array}$ \\
\hline $\begin{array}{c}\text { Vazão estimada a partir do uso } \\
\text { da terra }\end{array}$ & & & & & & \\
$\begin{array}{c}\text { X Vazão observada no posto da } \\
\text { ANA }\end{array}$ & 0,9991 & 0,9969 & $-0,5898$ & 1,4476 & 0,9998 & 0,9989 \\
$\begin{array}{c}\text { Vazão estimada na estação do } \\
\text { INMET }\end{array}$ & & & & & & \\
$\begin{array}{c}\text { X Vazão observada no posto da } \\
\text { ANA }\end{array}$ & 0,8099 & 0,6556 & $-11,2448$ & 27,5994 & 0,8943 & 0,7243 \\
$\begin{array}{l}\text { R = Correlação de Pearson; } \mathrm{R}^{2}=\text { Coeficiente de determinação; EQM = Erro quadrático médio; REQM = } \\
\text { Raiz quadrada do EQM; Índice D = Índice de desempenho de Willmott; Índice C = Índice de desempenho }\end{array}$
\end{tabular}


de Camargo e Sentelhas. Org.: Dos autores, 2019.

Após a análise da correlação entre as variáveis, calculou-se o erro quadrático médio, assim como sua raiz, sendo este indicador importante para verificar a concordância entre as variáveis (WILLMOTT et. al., 1985). Quanto menor o EQM, maior a concordância entre as variáveis. Identificou-se erro quadrático médio de $-0,5898 \mathrm{~m}^{3} / \mathrm{s} / \mathrm{m}^{2}$ entre vazões observadas e estimadas, indicando subestimativa do método. Em uma situação ideal, o EQM deve ser próximo a 0 (OLIVEIRA, 2016).

Figura 3 - Análise de regressão entre vazão observada e vazão estimada considerando categorias de uso e cobertura da terra para o ano hidrológico 2018/2019.

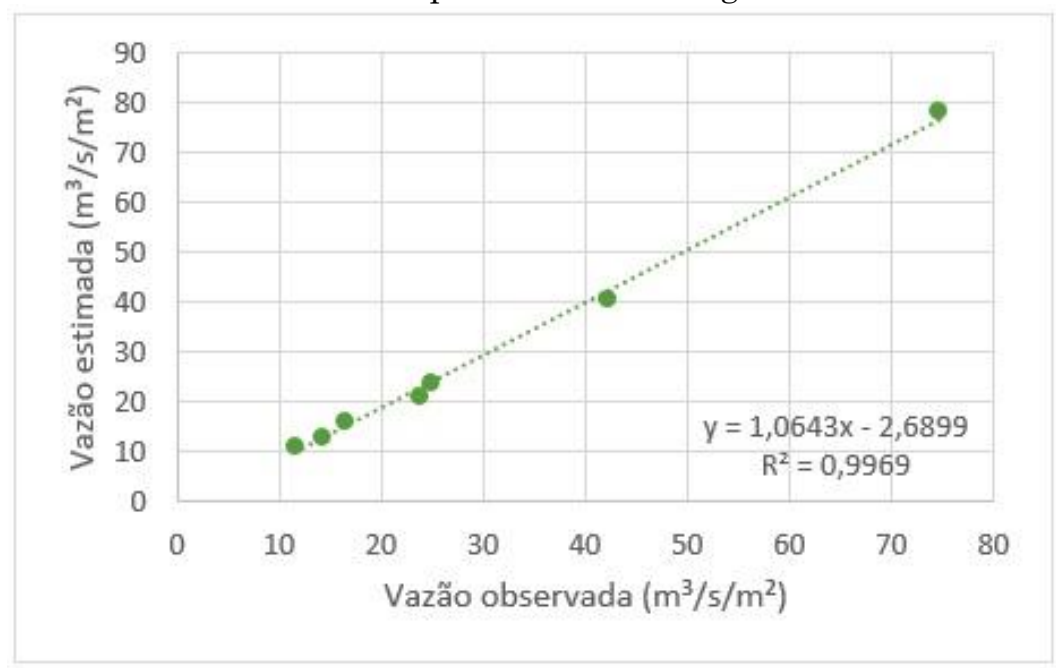

Org.: dos autores, 2019.

As vazões estimadas a partir das categorias de uso e cobertura da terra apresentaram índice $\mathrm{D}$ de 0,9998 e índice $\mathrm{C}$ de 0,9989, indicando desempenho ótimo do modelo SEBAL para ser utilizado na estimativa de vazão anual pelo balanço hídrico simplificado. Já para a estimativa realizada a partir da estação automática do INMET os índices de desempenho foram considerados ótimo (índice D igual a 0,8943) e bom (índice C igual a 0,7243). Apesar de extrapolar os valores, sobretudo no decorrer do período seco, o modelo também demonstrou desempenho satisfatório para estimativa de evapotranspiração a partir de dados mensais.

Com a validação da proposta de estimativa de vazão a partir do uso e cobertura da terra, aplicou-se a metodologia para a Bacia do Rio Bonito, tributária da bacia do Rio Araguaia, situada nos municípios de Caiapônia, Palestina de Goiás e Arenópolis e com área de aproximadamente $2,015 \mathrm{~km}^{2}$. A precipitação pluviométrica registrada entre 01/10/2018 e $30 / 09 / 2019$ foi de $1519,1 \mathrm{~mm}$, valor dentro da média considerada normal para a região. A ETo calculada foi de 2000,9 mm, com ETr correspondendo a $70 \%$ desse volume $(1378,3 \mathrm{~mm})$ e, considerando as categorias de uso e cobertura da terra, foi calculada ETr de 1175,9 mm.

A partir dos valores mensais e anual de $\mathrm{P}$ ETr foi estimada uma vazão média anual de 9,25 $\mathrm{m}^{3} / \mathrm{s} / \mathrm{m}^{2}$, variando de $226,7 \mathrm{~m}^{3} / \mathrm{s} / \mathrm{m}^{2}$ em março a $95,2 \mathrm{~m}^{3} / \mathrm{s} / \mathrm{m}^{2}$ em agosto, reforçando o efeito da sazonalidade sobre as estimativas mensais. Quando observados os valores estimados a partir das categorias de uso e cobertura da terra, que demonstraram correlação de 0,9991 para bacias com observações registradas em campo, a vazão anual estimada foi de $21,85 \mathrm{~m}^{3} / \mathrm{s} / \mathrm{m}^{2}$, a qual considera-se estar muito próxima aos valores reais se comparadas às vazões estimadas para a bacia do Rio Doce com as mesmas dimensões territoriais e volume de precipitação semelhante no período.

\section{CONSIDERAÇÕES FINAIS}

Frente aos valores apresentados pelos índices de desempenho do modelo, considera-se que o SEBAL é adequado para estimativa de vazão anual a partir de produtos de sensoriamento remoto considerando as categorias de uso e cobertura da terra e utilizando o balanço hídrico simplificado. Quando consideradas as estimativas mensais para valores observados nas estações meteorológicas automáticas do INMET, apesar da forte correlação com as vazões observadas, o desempenho é bom para as vazões 
anuais, mas estas apresentam erros médios significativos provocados pela sazonalidade dos cerrados, sendo que o período de estiagem de mais de 5 meses gera valores de déficit hídrico significativos.

Apesar de necessitar de mais testes, nota-se uma tendência a partir da equação de regressão de que os valores estimados devem seguir o mesmo padrão dos observados, atestando a validade do modelo para uso em bacias hidrográficas sem postos de monitoramento de vazão.

\section{AGRADECIMENTOS}

O presente trabalho foi realizado com apoio do Programa Nacional de Cooperação Acadêmica (PROCAD) da Coordenação de Aperfeiçoamento de Pessoal de Nível Superior - CAPES/Brasil Edital CAPES 071/2013 - Processo número 88881.068465/2014-01.

\section{REFERÊNCIAS}

ANA - Agência Nacional de Águas. Hidroweb Sistema de Informações Hidrológicas. Disponível em: < http://www.snirh.gov.br/hidroweb/publico/medi coes_historicas_abas.jsf\#>. Acesso em 15 de janeiro de 2020.

AHMAD, M.-UD-DIN, BASTIAANSSEN, W. G. M. Retrieving soil moisture storage in the unsaturated zone using satellite imagery and bi-annual phreatic surface fluctuations. Irrigation and Drainage Systems. v. 17, p. $141 \quad-\quad 161.2003$. https://doi.org/10.1023/A:1025101217521

ALLEN, R. G.; PEREIRA, L. S.; RAES, D.; SMITH, M. Crop evapotranspiration Guidelines for computing crop water requirements. In: FAO Irrigation and drainage paper 56. FAO, v.300, n.9, p. 1-297, 1998.

ALLEN, R. G. et al. SEBAL (Surface Energy Balance Algorithms for Land) advanced training and users manual - Idaho implementation. Idaho: Idaho University, USA, 2002. 98p.

ALLEN, R. G. et al. Satellite-Based Energy Balance for Mapping Evapotranspiration with Internalized Calibration (METRIC) - Model. In: Journal of irrigation and drainage engineering, v. 133, n.04, p.380-394, 2007.
https://doi.org/10.1061/(ASCE)0733-

9437(2007)133:4(380)

ALVES, W. S. Geotecnologias aplicadas em estudos hidrogeográficos na bacia do Rio Verdinho - Sudoeste de Goiás - Brasil. 209 f. Tese (Doutorado em Geografia) - Programa de Pós-Graduação em Geografia, Universidade Federal de Goiás - Regional Jataí, Jataí - GO, 2019.

ARIZA, A. Descripción y Corrección de Productos Landsat 8 LDCM (Landsat Data Continuity Mission). Bogotá: Instituto Geográfico Agustín Codazzi, 2013. 46p.

AYENEW, T. Evapotranspiration estimation using thematic mapper spectral satellite data in the Ethiopian rift and adjacent highlands. Journal of Hydrology, v.279, pp. 83 - 93. $2003 . \quad$ https://doi.org/10.1016/S00221694(03)00173-2

BASTIAANSSEN, W. G. M., Regionalization of surface flux densities and moisture indicators in composite terrain, Tese (Ph.D.), Wageningem Agricultural University, Wageningen: Netherlands, 273f, 1995.

BASTIAANSSEN, W. G. M. et al. A remote sensing surface energy balance algorithm for land (SEBAL): 2. Validation. Journal of Hydrology, v. 212 - 213, pp. 213 - 229. 1998. https://doi.org/10.1016/S0022-1694(98)00254-6

BASTIAANSSEN, W. G. M. SEBAL-based sensible and latent heat flux in the irrigated Gediz basin, Turkey. Journal of Hydrology, v. $229, \quad$ pp. $87-100.2000$. https://doi.org/10.1016/S0022-1694(99)00202-4

BEZERRA, B. G; SILVA, B. B.; FERREIRA, N. J. Estimativa da evapotranspiração real diária utilizando-se imagens digitais TM-Landsat5. Revista Brasileira de Meteorologia, vol.23, n.3, p.305-317, 2008. https://doi.org/10.1590/S010277862008000300005

CAMARGO, A. P.; SENTELHAS, P.C. Avaliação do desempenho de diferentes métodos de estimativa de evapotranspiração potencial no Estado de São Paulo, Brasil. In: Revista Brasileira de Agrometeorologia, v.5, n.01, p.89-97, 1997.

CUNHA, A. P. M. A.; ALVALÁ, R. C. S.; OLIVEIRA, G. S. Impactos das mudanças de cobertura vegetal nos processos de superfície na região semiárida do Brasil. Revista Brasileira de Meteorologia, v. 28, n.02, p.139-152. 2013. https://doi.org/10.1590/S010277862013000200003

ESRI - Environmental Systems Research Institute INC. ArcGis versão 10.6.1. EUA: Environmental Systems Research Institute, 
2018.

FREITAS, P. A. S. et al. Evapotranspiração de referência diária por diferentes modelos na bacia hidrográfica do Rio Capibaribe (Pernambuco - Brasil). In: Revista brasileira de meio ambiente, v.04, n.01, p. 35-45, 2018.

GIACOMONI, H .M.; MENDES, C. A. B. Estimativa de Evapotranspiração Regional por meio de Técnicas de Sensoriamento Remoto Integradas a Modelo de Balanço de Energia. Revista Brasileira de Recursos Hídricos, vol 13, n. 4, p. 33-42, out/dez 2008. https://doi.org/10.21168/rbrh.v13n4.p33-42

GOMES, H. B. Balanço de Radiação e energia em áreas de cultivo de cana-deaçúcar e cerrado no estado de São Paulo mediante imagens orbitais. Tese (Doutorado em Meteorologia) - Universidade Federal de Campina Grande, 2009. 108p.

HEMAKUMARA, H. M. et al. Evapotranspiration fluxes over mixed vegetation areas measured from large aperture scintillometer. In Agricultural Water Management, v. 58, p. 109-122. 2003. https://doi.org/10.1016/S0378-3774(02)00131-2

IBGE - Instituto Brasileiro de Geografia e Estatística. Base Cartográfica - 2014. Disponível em: <http://www.ibge.gov.br>. Acesso em 15 de setembro de 2019.

INMET - Instituto Nacional De Meteorologia Rede de estações automáticas. Disponível em

http://www.inmet.gov.br/portal/index.php?r=es tacoes/estacoesAutomaticas $>$ Acesso em 10 out. 2019.

INPE - Instituto Nacional De Pesquisas Espaciais. Projeto PRODES de monitoramento do Cerrado brasileiro por satélite. Disponível em: $<$ http://terrabrasilis.dpi.inpe.br/app/dashboard/ deforestation/biomes/cerrado/increments $>$.

Acesso em 15 de Janeiro de 2020.

KIMURA, R. et al. Evapotranspiration estimation over the river basin of the Loess Plateau of China base on remote sensing, Journal of Arid Environments, v. 68, p. 5365. 2007.

https://doi.org/10.1016/j.jaridenv.2006.03.029

KLINK, C. A.; MACHADO, R. B. A conservação do Cerrado Brasileiro. In: Megadiversidade, v.01, n.01, p. 147-155, 2005.

KONGO, V. M., JEWITT, G. P. W. Preliminary investigation of catchment hydrology in response to agricultural water use innovations: A case study of the Potshini catchement South Africa. Physics and Chemistry of the Earth, v. 31, p.976-987. 2006. https://doi.org/10.1016/j.pce.2006.08.014

LIMA, W. P. A silvicultura e a água: ciência, dogma e desafios. Cadernos do diálogo 1, 2010, p.1-68. Disponível em: <https://www.conservation.org/brasil/publicaco es/

Documents/cadernos_do_dialogo_volume_1_ag ua_e_silvicultura.pdf $>$ Acesso em 15 de janeiro de 2020.

LI, S.; WHENZI, Z. Satellite-based actual evapotranspiration estimation in the middle reach of the Heihe River Basin using the SEBAL method. In: hydrological processes, v.24, p.3337-3344, 2010. https://doi.org/10.1002/hyp.7748

LIOU, Y.; KAR, S. K. Evapotranspiration Estimation with Remote Sensing and Various Surface Energy Balance Algorithms-A Review. In: Energies, v.7, p.2821-2849, 2014. https://doi.org/10.3390/en7052821

MACHADO, R. B. et al. Estimativa de perda da área do Cerrado Brasileiro. Relatório técnico não publicado. Brasília-DF: Conservação internacional, 2004. 25p.

MARIANO, Z. F. A importância da variável climática na produtividade de soja no sudoeste de Goiás. 253 f. Tese (Doutorado em Geografia) - Programa de Pós-Graduação em Geografia, Universidade Estadual de São Paulo, Rio Claro, 2005.

MARTINS, A. P. Uso de dados do sensor MODIS/AQUA e do algoritmo SEBAL para estimativa da evapotranspiração real na bacia do Rio Paranaíba. 150 f. Tese (Doutorado em Geografia) - Programa de PósGraduação em Geografia, Universidade Federal de Uberlândia, Uberlândia - MG, 2015. MARTINS, A. P.; GOMES FILHO, R. R. Estudo e Gestão de Bacias Hidrográficas. In: GOMES FILHO, R. R. (org.) Gestão de recursos hídricos: conceitos e experiências em bacias hidrográficas. Goiânia, GO: Editora da UEG, 2013. p.11-34.

MARTINS, A. P.; SCOPEL, I.; SOUSA, M. S.; PEIXINHO, D.M. Uso da terra e cobertura vegetal de 1985 a 2015 no Sudoeste de Goiás e relações com o meio físico. In: PEIXINHO, D.; SOUSA, M.S. (Orgs.). Reconfiguração do Cerrado: uso, conflitos e impactos ambientais. Goiânia, GO: Gráfica UFG, 2016. p. 11-34.

MENDONÇA, J. C. Estimação da evapotranspiração regional utilizando imagens digitais orbitais na região Norte Fluminense, RJ. Tese. Universidade Estadual do Norte Fluminense, Campos dos Goytacazes, RJ, Brasil. 2007.

NICÁCIO, R. M. Evapotranspiração real e 
umidade do solo usando dados de sensores orbitais e a metodologia SEBAL na bacia do Rio São Francisco. 2008. 337f. Tese (Doutorado em Engenharia Civil) Programa de Pós-Graduação em Engenharia Civil, Universidade Federal do Rio de Janeiro, 2008.

OLIVEIRA, E. A. Métodos para análise de concordância: estudo de simulação e aplicação a dados de evapotranspiração. 177 f. Tese (Doutorado em Ciências) - Escola Superior de Agricultura "Luiz de Queiroz", Universidade de São Paulo, Piracicaba, 2016.

PEREIRA, A. R.; SEDIYAMA, G. C.; VILLA NOVA, N. A. Evapotranspiração. Campinas:Fundag, 2013. 323 p.

REBOUÇAS, A. C. Panorama da água doce no Brasil. In: REBOUÇAS, A. C. (org) Panoramas da degradação do ar, da água doce e da terra no Brasil. São Paulo: IEA/USP, 1997. $150 \mathrm{p}$.

ROCHA, G. F. et al. Deteç̧ão de desmatamentos no bioma Cerrado entre 2002 e 2009: padrões, tendências e impactos. In: Revista Brasileira de Cartografia, v. 03, n.63, p.341-349, 2011.

RUHOFF, A. L. Sensoriamento Remoto aplicado à estimativa de evapotranspiração em biomas tropicais. 1850 f. Tese (Doutorado em Recursos Hídricos e Saneamento Ambiental) - Instituto de Pesquisas Hidráulicas, Universidade Federal do Rio Grande do Sul, Porto Alegre, 2011.

SANO, E. E. et al. Land cover mapping of the tropical savanna region in Brazil. In: Environmental Monitoring and Assessment (Print), v. 166, p. 113-124, 2010. https://doi.org/10.1007/s10661-009-0988-4

SIEG - Sistema Estadual de Estatística e Informações Geográficas de Goiás. Base cartográfica [2012]. Disponível em: $<$ http://www.sieg.go.gov.br>. Acesso em 15 de setembro de 2019

SUN, Z. et. al. Evapotranspiration estimation based on the SEBAL model in the Nansi Lake Wetland of China. In: Mathematical and Computer Modelling, v.54, p.1086-1092, 2011.

https://doi.org/10.1016/j.mcm.2010.11.039

TASUMI, M. et al. Operational aspects of satellitebased energy balance models for irrigated crops in the semi-arid U.S. Irrigation and Drainage Systems, v. 19, p. 355-376. 2005. https://doi.org/10.1007/s10795005-8138-9

USGS - United States Geological Survey. Download de imagens Landsat, SRTM e Sentinel. Disponível em: < https://earthexplorer.usgs.gov/>. Acesso em 15 de setembro de 2019

WILLMOTT, C. J. et al. Statistics for the evaluation of model performance. In: Journal of Geophysical Research, v.90, n.C5, p.8998-9005, 1985. 